\title{
2 Forschungsmethodik, Relevanz der Arbeit für die Psychotherapiewissenschaften und Forschungsstand
}

\subsection{Forschungsmethodik}

Ich verfolge in dieser Arbeit eine psychoanalytische Herangehensweise, indem ich mit den spezifischen Instrumenten der Psychoanalyse und der Hermeneutik ${ }^{4}$ nach den bewussten und unbewussten Faktoren im Erleben, Verstehen und in der Verarbeitung der Finanzkrise bei einzelnen Individuen und Gruppen forsche. $\mathrm{Zu}$ diesem Zweck wurden qualitative Interviews mit Menschen aus Bereichen geführt, die in ihrer Arbeit mit den Auswirkungen der Finanzkrise in Berührung kamen; es sind Unternehmer und Unternehmensberater im weiteren Sinn, die, vereinfacht gesagt, die Bereiche der Investition und Regulation repräsentieren.

Als Basis für die Analyse dieser Interviews war eine umfassende Literaturauswertung notwendig, die die theoretischen und begrifflichen Voraussetzungen klärte und eine historische Einordnung des Krisenereignisses ermöglichte.

Thematisch ist die Arbeit an der Schnittstelle angesiedelt, wo sich die Psychoanalyse als Theorie des psychischen Erlebens mit der Psychopathologie und der Sozialpsychologie trifft.

Pritz und Teufelhardt haben Psychotherapie als „Wissenschaft vom Subjektiven“ in dem Sinn bezeichnet, in dem die subjektive Erfahrung im psychotherapeutischen Setting einer (Selbst-)Reflexion unterzogen wird und im Austausch mit dem therapeutischen Gegenüber Intersubjektivität miteinschließt; beide, Selbstreflexion und Beziehungserleben, tragen Veränderungspotenzial in sich (vgl. Pritz \& Teufelhart, 1996).

4 In der philosophischen Hermeneutik geht es darum, „,aß die Methode der Interpretation und des Verstehens, wie wir sie in den Geisteswissenschaften vorfinden, einen sui-generis-Charakter habe und daß diese Methode auf keine Weise auf naturwissenschaftliche Verfahren zurückführbar sei“" (Stegmüller, 1979, S. 107).

Hermeneutik wird im Sinne Wilhelm Diltheys als Kunst der Interpretation aufgefasst (vgl. Stegmüller, 1979, S. 106). „Den lebendigen Menschen aus ihm selbst zu verstehen, war das Bestreben Diltheys. Die Möglichkeit solcher Auslegung sah er in der Auffassung des Menschen als eines Gliedes der geschichtlich-gesellschaftlichen Welt, seine Einbettung in den Werde- und Wirkzusammenhang des historischen Ablaufs, verbunden mit einer historischen Relativierung der Weltauffassungen“" (Stegmüller, 1978, S. 151). 
Die in den Interviews hervortretende Subjektivität steht im Fokus der empirisch-qualitativen Untersuchung im Rahmen dieser Arbeit; die Äußerungen dieser „Subjekte“, ihre Modelle und Interpretationen der Wirklichkeit können, wie es Zielsetzung dieser Arbeit ist, vor dem Hintergrund der Gesellschaft, in der sie leben, analysiert werden. Je nach dem Blickwinkel der Forscherin tritt entweder das Subjekt oder die Gesellschaft in den Vordergrund. Ohne einen induktiven Schluss im Sinne einer Verallgemeinerung einzelner Aussagen der Befragten ziehen zu wollen, ist es doch interessant, welche dieser Erfahrungen von den Interviewpartnern geteilt werden bzw. welche Unterschiede bestehen. Dazu gehört auch die Analyse der Interpretationsmuster, wie Krisenerfahrungen subjektiv eingeordnet werden.

So wird die Psychoanalyse sowohl bei den Interviews als auch bei der umfassenden Literaturanalyse, die das aktuelle Krisengeschehen und die Auswirkungen der Weltwirtschaftskrise in den 30er-Jahren des letzten Jahrhunderts thematisch behandelt, in ihrer kultur-, gesellschafts- und ideologiekritischen Funktion eingesetzt.

„Wie das Licht des Bewußtseins nur einen Teil der psychischen Welt erhellt, die im Dämmer des Vor- und in der Nacht des Unbewußten liegt, so liegt auch für die Teilhaber der Kultur ein gut Teil ihrer sozialen Welt unter dem Horizont. Das Dunkel falschen Bewusstseins verbirgt Struktur und Dynamik der Lebens- und Sozialgeschichte. In dem Maße, wie sie die wirklichen Bedingungen ihrer Praxis nicht kennen, sind die Individuen und Kollektive deren nicht Herr, unterliegen sie der "List' einer Vernunft oder Unvernunft, die nicht die ihre ist" (Dahmer, 2013a, S. 379).

Als Psychoanalytikerin und Nichtökonomin habe ich mit dieser Themenstellung mir unbekanntes Terrain betreten. Es kommt zu Überschneidungen mit wirtschafts- und sozialwissenschaftlichen Fragestellungen, die für mich im Sinne einer Interdisziplinarität, aber auch durch den Kontakt mit meinen Interviewpartnern einen erheblichen „Mehrwert" an Wissen und Erfahrung gebracht haben. In der Zusammenarbeit bisher getrennter Wissenschaftsdisziplinen könnten in Zukunft durch die „Fremdheit“ des jeweils anderen Gebiets immanente „blinde Flecken“ "besser ausgeleuchtet werden und könnte ein befruchtender Dialog entstehen.

\subsection{Relevanz der Arbeit für die Psychotherapiewissenschaften}

Die Relevanz dieser Arbeit für die Psychotherapiewissenschaften ist mehrfach begründet. Einerseits über die Erforschung der durch die ökonomische Krise bedingten psychischen Leiden und materiellen Verluste, die wegen ihres Aus- 
maßes nicht nur einzelne Subjekte, sondern gesellschaftliche Gruppen und größere Einheiten wie Nationen betreffen.

Indem ich nach den Repräsentationen des Unbewussten frage, in denen sich die Krise manifestiert, wird die Ebene des scheinbar Gegebenen überschritten, kritisch infrage gestellt und eine Veränderungsmöglichkeit im Hinblick auf die eigene Lebensgeschichte, aber auch im Hinblick auf die ökonomische Bedingtheit der sozialen Welt aufgetan und beider Historizität dargelegt.

Damit folge ich dem Grundprinzip der Psychoanalyse, Unbewusstes bewusst zu machen. Die Reflexion der Erklärungsmodelle von Krisen und ihrer Lösung kann in Bezug auf das in ihnen nicht zur Sprache Gekommene, das heißt, das Verdrängte und Verleugnete, untersucht werden und so zu einem besseren Verständnis von sozialen Prozessen führen, die gemeinhin nicht im Fokus der Psychoanalyse stehen und beforscht werden. Durch das Eingebettetsein der Forschungsfrage in soziologische, historische, ökonomische und politische Fragestellungen wird ein Dialog zwischen den Psychotherapiewissenschaften und anderen Wissenschaften eröffnet.

\subsection{Forschungsstand}

Zur Finanzkrise und ihren Folgen liegt eine große Anzahl von Publikationen im Bereich der Ökonomik vor. Als Nichtökonomin habe ich mich auf jene Arbeiten gestützt, die die Entstehung und die Folgen der Finanzkrise 2008 in einen $\mathrm{Zu}$ sammenhang mit veränderten Parametern in der Wirtschaftspolitik der betroffenen Staaten gebracht und diese gemeinsam mit den zugrundeliegenden Paradigmen dargestellt und einer Kritik zugänglich gemacht haben (Hetzer, 2011; Piketty, 2015; Sandel, 2012; Stiglitz, 30.6.2015, 2004, 2016).

Gibt man in Google den Begriff „Finanzkrise 2008“ ein $^{5}$, werden 2.710.000 Ergebnisse (0,35 seconds) angezeigt.

Bücher wie „Die Abstiegsgesellschaft. Über das Aufbegehren in der regressiven Moderne“ (Nachtwey, 2017) oder der Sammelband „Die große Regression" (Geiselberger, 2017), in dem Autoren unterschiedlicher Disziplinen zu Wort kommen, analysieren die Auswirkungen eines neoliberalen Wirtschaftssystems in ökonomischer, sozialer und politischer Hinsicht; ${ }^{6}$ sie weisen auf dessen desta-

$5 \quad 11.12 .2017$.

6 Siehe dazu auch Bianchi (2009), Foster (2016), Grubner (2017), Marlock (2010), Modena (2010), Ottomeyer (2010), Schuch (2010) oder Strenger (2017). Für meine Arbeit sind auch jene Publikationen relevant, die Veränderungen im Hinblick auf Identität und soziales Verhalten aus einem psychoanalytischen Blickwinkel beleuchten (Verhaeghe, 2013, 2016). 
bilisierende und demokratiegefährdende Tendenzen hin. Schulden, Arbeitslosigkeit, wachsende Armut und fehlende Perspektiven der Veränderung führen durch die Globalisierung zu Migrationsbewegungen, die Krisen nicht mehr als regional begrenztes Phänomen erscheinen lassen und neben einzelnen Personen auch Gruppen und Staaten betreffen. Dazu gehören jene Entwicklungen, die sich in kritischer Weise mit dem Resilienzdiskurs auseinandersetzen, der das Individuum und seine Veränderbarkeit, das heißt Anpassungsfähigkeit im Hinblick auf Krisen, in den Vordergrund stellt (Gebauer, 2017; Merk, 2017; Neocleous, 2017; Ottomeyer, 2010, 2014; Ottomeyer \& Reddemann, 2017).

In den Jahren nach der Krise wurden zahlreiche Beiträge veröffentlicht, in denen sich die Autoren mit dem Phänomen der Krise aus psychoanalytischer, sozialpsychologischer und soziologischer $\operatorname{Sicht}^{7}$ auseinandergesetzt haben. Thematisch kann man sie folgendermaßen gliedern: solche, die sich mit der Rolle des Geldes und seiner Funktion auseinandersetzen (Decker, Türcke \& Grave, 2011; Focke, Kaiser \& Scheferling, 2013; Vinnai, 2013) und damit einer Tradition in der Psychoanalyse folgen (Abraham, 1917; Borneman, 1977; Mauss, 2015; Simmel, 1989), was für die vorliegende Arbeit jedoch nicht im Vordergrund steht; und solche, die analysieren, wie die Finanzmärkte aus einer psychologischen oder psychoanalytischen Sicht funktionieren und aufzeigen, dass wirtschaftliches Handeln nur scheinbar rationalen Prinzipien folgt.

Ängste und Gruppendenken spielen eine viel größere Rolle als angenommen. Sehr oft wird auf das Konzept des Narzissmus bei der Analyse der innerpsychischen Prozesse, die den Handel mit spekulativen Finanzprodukten charakterisieren, zurückgegriffen (Bianchi, 2009; Patsiaouras, Fitchett \& Davies, 2015; Shulman, 2016; Sievers, 2010, 2011). ${ }^{8}$

Akerlof und Shiller gehen in ihrem Buch „Animal Spirits“ (2009) in Abweichung von Adam Smiths Konzept eines Marktes, der sich im Idealzustand (das heißt ohne staatliche Eingriffe auf der Basis von rationalen ökonomischen Entscheidungen seiner Subjekte) selbst reguliert, davon aus, dass sogenannte Animal Spirits ${ }^{9}$, zu denen sie etwa Vertrauen, Fairness, Korruption, Geldillu-

7 Siehe dazu das Schwerpunkthema „Triebökonomie und Krise des Kapitalismus“ in der Zeitschrift psychosozial, Nr. 129, aus dem Jahr 2012.

8 Kirsner (1990) hat bereits 1990 eine solche Analyse, bezogen auf den Börsenkrach 1987, vorgelegt.

9 „Der Begriff Animal Spirits entstammt dem lateinischen spiritus animalis. In seiner ursprünglichen Verwendung in der Antike und später im Mittelalter bedeutet das Wort animalis, geistig' oder ,belebend'. Damit verweist es auf eine elementare gedankliche Energie und Antriebskraft" (Akerlof \& Shiller, 2009, S. 21). 
sion $^{10}$ und Geschichten ${ }^{11}$ zählen, eine viel größere Rolle spielen, als von den Theoretikern einer klassischen Wirtschaftstheorie ${ }^{12}$ angenommen wird (vgl. Akerlof \& Shiller, 2009, S. 24). Einzelne dieser Animal Spirits werden auch im Rahmen der Analyse der Interviews eine Rolle spielen. ${ }^{13}$ Der Begriff geht auf den Ökonomen Maynard Keynes zurück, der im Jahr 1936 sein Werk „Allgemeine Theorie der Beschäftigung, des Zinses und des Geldes“ veröffentlicht hat (vgl. Akerlof \& Shiller, 2009, S. 8). „Nach Keynes sind die Animal Spirits die wichtigste Ursache für Schwankungen der Konjunktur und für unfreiwillige Arbeitslosigkeit" (Akerlof \& Shiller, 2009, S. 10).

10 Unter Geldillusion versteht man, dass von Nominalwerten statt von Realwerten des Geldes ausgegangen wird, das heißt, dass Lohnverluste durch Inflation bei Lohnverhandlungen etwa nicht in Rechnung gestellt werden. „Nicht nur Lohnvereinbarungen beziehen sich in den meisten Fällen auf Nominalwerte, sind also nicht indexiert. Dasselbe gilt in der Regel auch für Finanzgeschäfte. Verträge zwischen Schuldnern und Gläubigen sind fast nie indexiert" (Akerlof \& Shiller, 2009, S. 80 f.).

11 „Von besonderer Bedeutung sind Geschichten eines neuen Zeitalters, die vorgeben, einen historischen Wandel zu beschreiben, der die Wirtschaft grundlegend verändert" (Akerlof \& Shiller, 2009, S. 89). „Die Geschichten von jungen Leuten, die mit dem Internet ein Vermögen machten, waren Neuauflagen der Geschichten aus der Zeit des Goldrauschs im 19. Jahrhundert [...] Ein Klima der Zuversicht geht tendenziell mit inspirierenden Geschichten einher, mit Geschichten über neue geschäftliche Initiativen und über die Art und Weise, wie andere zu Reichtum gelangen. In der Vergangenheit wurden die großen Aufschwünge an den Börsen überall auf der Welt meist von Geschichten über ein ,neues Zeitalter" begleitet“" (Akerlof \& Shiller, 2009, S. 90).

In diesem Zusammenhang stellt sich die Frage, inwiefern diese Geschichten, wenn sie mit negativen Erinnerungen gepaart sind, nicht auch als kollektive Traumata verstanden werden können. In Anspielung auf den Bankensturm im Jahr 1893 in den USA schreiben die Autoren: „Die Bankpanik explodierte und wurde zu einer gesellschaftlichen Epidemie, die Menschen reagierten auf die langen Schlangen, die sie vor den Banken sahen, ließen sich von der Angst dieser Leute anstecken und erzählten erneut Geschichten über frühere Bankpaniken“ (Akerlof \& Shiller, 2009, S. 98).

12 „Die traditionelle Theorie sagt uns, dass kapitalistische Systeme mit freien Märkten in ihrem Kern vollkommen und stabil seien. Staatliche Eingriffe seien nur selten notwendig. Im Gegenteil, solche Eingriffe seien sogar potenziell schädlich. Besteht die Gefahr einer bedeutenden Depression, so geht diese einzig und allein von Interventionen des Staates aus“ (Akerlof \& Shiller, 2009, S. 18).

13 „Unsere Sichtweisen sind eng mit unserer eigenen Lebensgeschichte und den Lebensgeschichten anderer verwoben. Fasst man all diese individuellen Geschichten zusammen, so erhält man die Geschichte eines Landes oder der Beziehung zwischen verschiedenen Ländern, die ihrerseits eine wichtige Rolle im Wirtschaftsgeschehen spielt" (Akerlof \& Shiller, 2009, S. 24). 
Tucketts (2013) Buch ist mit seiner psychoanalytischen, auf den Theorien Kleins und Bions basierenden fundierten Analyse der Krise von 2008 einzigartig in der Verbindung dieser Wissenschaftsdisziplinen geblieben und hat mir indirekt den Weg gewiesen, mich mit dem Phänomen der Krise in einer Form auseinanderzusetzen, die - im Unterschied zu Tuckett - mehr den kulturkritischen und metapsychologischen Schriften der Psychoanalyse und der Gruppenanalyse folgt und normale Akteure im Wirtschaftsleben ins Zentrum der Untersuchung rückt. ${ }^{14}$

Eine der jüngsten Publikationen, die sich mit dem kulturpsychologischen Aspekt der Finanzkrise 2008 auseinandersetzt, ist jene von Sandner (2018 [!]). Der Autor macht das patriarchale Gesellschaftsmodell von der Urhorde, das Freud in „Totem und Tabu“ beschreibt und das Sandner auf die heutige Gesellschaft überträgt, als wesentliche Ursache für die Finanzkrise fest. ${ }^{15}$

„War es früher der militärisch mächtigste Fürst, dann die mächtigste Industriegruppe, so ist es heute die mächtigste Finanzgruppe oder die Finanzwirtschaft insgesamt, welche die Position des Hordenvaters innehat, wenn es ihr gestattet ist, die Macht zu gewinnen und auszuüben " (Sandner, 2018 [!], S. 15).

Diese Erklärungsweise erscheint mir für das Verständnis des Geschehens nur zum Teil erhellend. Sandner bezieht sich mit dem Begriff der „Bordell-Ökonomie", der den Finanzkapitalismus charakterisieren soll, auf ein anderes Buch zur Finanzkrise, das die „Dämonen des Kapitals“ im Titel trägt und verspricht, die „Ökonomie auf Freuds Couch“ zu legen (Sedlacek \& Tanzer, 2015). Personifizierungen des ökonomischen Geschehens, das Unterfangen, der Ökonomie eine Seele zuzuweisen (vgl. Sedlacek \& Tanzer, 2015, S. 15), pathologische Verhaltensmuster vom Individuum auf die Gesellschaft zu übertragen und eine willkürliche Zuordnung psychoanalytischer Begriffe und Mythen $\mathrm{zu}$ wirtschaftlichen Vorgängen vorzunehmen, sind einem tieferen Verständnis gesellschaftlicher

14 Die Basis für Tucketts Theorie der „emotionalen Finanzwirtschaft“ bilden Interviews, die er 2007 mit 52 Vermögensverwaltern, die schon zehn Jahre oder länger in ihrer Funktion in großen Finanzzentren der Welt tätig waren und mindestens eine Milliarde Dollar verwalteten, geführt hat (vgl. Tuckett, 2013, S. 61).

15 „Wenn nicht alles trügt, entspricht der geschilderte ökonomische und finanzkapitalistische Prozess genau dem ,Hordenmodell` von Freud: Wenn man die Dynamik des Finanzkapitalismus entgrenzt, grenzenlos werden lässt, geschieht das, was geschehen ist: Er wird übermächtig, zur einzig und allein dominierenden Macht, die nicht nur die wirtschaftlichen und politischen Prozesse bestimmt, sondern schlicht das gesamte Leben der Menschen auf der ganzen Welt mit der Gefahr der Zerstörung der wirtschaftlichen und gesellschaftlichen Grundlagen der Staaten und der in ihnen lebenden Menschen“ (Sandner, 2018 [!], S. 153). 
Prozesse jedoch genauso abträglich wie einer Annäherung der beiden Wissenschaften Psychoanalyse und Ökonomik.

In einigen Publikationen spielt das Konzept der Gier eine große Rolle, das aus verschiedenen Blickwinkeln beleuchtet wird (Rothschild, 2010; Sievers, 2011; Wirth, 2010). Darauf wird in den Analysen der Interviews Bezug genommen werden.

$\mathrm{Zu}$ den Auswirkungen der Krise auf die psychische Gesundheit gibt es eine Reihe von wissenschaftlichen Publikationen im europäischen Kontext: solche, die länderübergreifend sind, wie zum Beispiel die umfassende Literatur-Review von Martin-Carrasco et al. (2016), die 354 Arbeiten umfasst, oder solche, die länderspezifisch ausgerichtet sind (Berghofer \& Psota, 2013; Bundesministerium für Arbeit, 2017; Christodoulou, 2017; Moreno-Küstner \& Gutierrez, 2017; Ruggeri \& Tomassi, 2017), um nur einige zu nennen. Daten und Fakten findet man auch bei der WHO (2011) und der OECD (2013, 2017).

Dornes (2016) kritisiert in seinem Buch die Hypothese, dass psychische Krankheiten im Kapitalismus zunehmen. Er bezieht sich auf (Längsschnitt-) Studien, die diese Annahme widerlegen, und argumentiert mit einer Zunahme an Diagnosen, die nicht unbedingt mit einer Zunahme an Erkrankungen einhergehen muss. Mein Fokus ist jedoch auf die zeitlich nahen Folgewirkungen der Wirtschaftskrise gerichtet und umfasst daher kürzere Zeiträume. ${ }^{16}$

In den Kapiteln, die sich mit den Konzepten des Unbewussten und der Psychoanalyse der Zwischenkriegszeit auseinandersetzen, beziehe ich mich auf die Werke Freuds und seiner Schüler, wie Bernfeld, Fenichel, Fromm oder Reich.

Das Konzept des sozialen Unbewussten wurzelt in den Anfängen der Psychoanalyse, wurde in den letzten Jahren in den englischsprachigen Ländern weiterentwickelt und stößt besonders im Bereich der Gruppenanalyse auf großes Interesse (Hopper, 2006; Hopper \& Weinberg, 2011, 2016, 2017; Raufman \& Weinberg, 2017).

16 Diese Studien klammert Dornes aus, wenn er sich auf die Zunahme von Depressionen bezieht: ,Von den drei [Studien], die einen Anstieg berichten, stammen zwei aus Griechenland und beziehen sich auf die Jahre 2008 und 2011. Lässt man diese beiden Untersuchungen wegen der besonderen Umstände in diesem Land und Zeitraum unberücksichtigt, so ist die Befundlage vollständig ausbalanciert (keine Veränderung in vier Untersuchungen sowie je eine Zu- und Abnahme)“ (Dornes, 2016, S. 17). Zum Teil wirkt Dornes' Argumentation zynisch, wie man in einer Anmerkung nachlesen kann: „Es ist nicht auszuschließen, dass die jüngste wirtschaftliche Krise in manchen besonders schwer betroffenen Länder [sic!] einen Effekt hat [...] Jedoch wird auch vom Gegenteil berichtet. Seit der Krise von 2008 leben beispielsweise die Isländer gesünder (unter anderem wegen gesunkener Einkommen)“ (Dornes, 2016, S. 130). 
Die kulturkritischen Aspekte der Psychoanalyse und deren Verleugnung, die wie ein roter Faden das Gewebe dieser Arbeit durchziehen, wurden vielfach beleuchtet (Jacoby, 1975, 1985). Eine der jüngsten Publikationen ist das Buch von Herzog (2017). Peglau (2013) hat vor ein paar Jahren ein Buch über Reich und die Psychoanalyse im Nationalsozialismus veröffentlicht, das wesentliche neue Fakten über den Umgang mit Reich und über die Bedingungen für das Weiterleben der Psychoanalyse unter den Nationalsozialisten zu Tage gebracht hat. In diesem Zusammenhang sind auch die Arbeiten von Dahmer zu nennen, der mit seinem kritischen Blick und Denken die gesellschaftskritische Funktion der Psychoanalyse einmahnt (Dahmer, 2013a, 2013b, 2013c).

Open Access Dieses Kapitel wird unter der Creative Commons Namensnennung 4.0 International Lizenz (http://creativecommons.org/licenses/by/4.0/deed.de) veröffentlicht, welche die Nutzung, Vervielfältigung, Bearbeitung, Verbreitung und Wiedergabe in jeglichem Medium und Format erlaubt, sofern Sie den/die ursprünglichen Autor(en) und die Quelle ordnungsgemäß nennen, einen Link zur Creative Commons Lizenz beifügen und angeben, ob Änderungen vorgenommen wurden.

Die in diesem Kapitel enthaltenen Bilder und sonstiges Drittmaterial unterliegen ebenfalls der genannten Creative Commons Lizenz, sofern sich aus der Abbildungslegende nichts anderes ergibt. Sofern das betreffende Material nicht unter der genannten Creative Commons Lizenz steht und die betreffende Handlung nicht nach gesetzlichen Vorschriften erlaubt ist, ist für die oben aufgeführten Weiterverwendungen des Materials die Einwilligung des jeweiligen Rechteinhabers einzuholen.

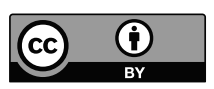

